\title{
On Boundary Damped Inhomogeneous Timoshenko Beams and Related Problems
}

\author{
R. Picard* \& B.A. Watson ${ }^{\dagger} \ddagger$
}

July 23, 2021

\begin{abstract}
We consider the model equations for the Timoshenko beam as a first order system in the framework of evolutionary equations as developed in [2]. The focus is on boundary damping, which is implemented as a dynamic boundary condition. A change of material laws allows to include a large class of cases of boundary damping. By choosing a particular material law, it is shown that the first order approach to Sturm-Liouville problems with boundary damping is also covered.
\end{abstract}

\section{Introduction}

The homogeneous Timoshenko beam model is given by a second order system of the form

$$
\begin{aligned}
\nu_{1} \partial_{0}^{2} \phi-\partial_{1} \kappa_{1}^{-1} \partial_{1} \phi+\kappa_{2}^{-1}\left(\partial_{1} u+\phi\right) & =f_{1} . \\
\nu_{2} \partial_{0}^{2} u-\partial_{1} \kappa_{2}^{-1}\left(\partial_{1} u+\phi\right)+d \partial_{0} u & =f_{2},
\end{aligned}
$$

Here $u$ denotes the radial displacement of the beam and $\phi$ its angular displacement. The real time independent parameters $\nu_{1}, \kappa_{1}, \nu_{2}, \kappa_{2}, d$, describe physical and geometrical properties of the beam, which, however, we will not elaborate upon further, since for the analysis their interpretation is not important. It may be interesting to note that these equations are the 1-dimensional version of the Reissner-Mindlin plate model, compare [3]. To reformulate (11) as a first order system we introduce the velocities $\eta:=\partial_{0} \phi$ and $s:=\partial_{0} u$ as new unknowns. Moreover, we let

$$
V_{2}:=\kappa_{2}^{-1}\left(\partial_{1} u+\phi\right)
$$

and

$$
V_{1}:=\kappa_{1}^{-1} \partial_{1} \phi .
$$

*Institut für Analysis,FR Mathematik, TU Dresden, D-01062 Dresden, Germany

${ }^{\dagger}$ School of Mathematics, University of the Witwatersrand, Johannesburg, South Africa.

${ }^{\ddagger}$ Funded in part by NRF grant IF2011032400120 and the Centre for Applicable Analysis and Number Theory 
Now (11) can be written as

$$
\begin{aligned}
& \nu_{1} \partial_{0} \eta-\partial_{1} V_{1}+V_{2}=f_{1} \\
& \nu_{2} \partial_{0} s-\partial_{1} V_{2}+d s=f_{2}
\end{aligned}
$$

together with the spatial differentiated equations (2) and (3) resulting in the temporal equations

$$
\begin{array}{r}
\partial_{0} \kappa_{1} V_{1}-\partial_{1} \eta=0 \\
\partial_{0} \kappa_{2} V_{2}-\partial_{1} s-\eta=0 .
\end{array}
$$

Written as a system we have

$$
\left(\partial_{0} \mathcal{M}_{0}+\mathcal{M}_{1}+\left(\begin{array}{cccc}
0 & -\partial_{1} & 0 & 0 \\
-\partial_{1} & 0 & 0 & 0 \\
0 & 0 & 0 & -\partial_{1} \\
0 & 0 & -\partial_{1} & 0
\end{array}\right)\right)\left(\begin{array}{c}
V_{1} \\
\eta \\
s \\
V_{2}
\end{array}\right)=\left(\begin{array}{c}
0 \\
f_{1} \\
f_{2} \\
0
\end{array}\right)
$$

where

$$
\mathcal{M}_{0}:=\left(\begin{array}{cccc}
\kappa_{1} & 0 & 0 & 0 \\
0 & \nu_{1} & 0 & 0 \\
0 & 0 & \nu_{2} & 0 \\
0 & 0 & 0 & \kappa_{2}
\end{array}\right), \mathcal{M}_{1}:=\left(\begin{array}{cccc}
0 & 0 & 0 & 0 \\
0 & 0 & 0 & 1 \\
0 & 0 & d & 0 \\
0 & -1 & 0 & 0
\end{array}\right)
$$

Here $\nu_{1}, \kappa_{1}, \nu_{2}, \kappa_{2}$ are positive real time independent parameters. It is essentially the latter form in which we will approach the issue of boundary damping, since it will allow us to utilize the framework of [2]. The structural features of the first order system allow us also to make - almost effortlessly - the transition to more general media.

\section{Functional Analytic Framework}

Key to the approach presented here is the closure of the differentiation operator on $C_{1}(\mathbb{R}, H)$ functions with compact support, i.e. acting on $\stackrel{C}{C}_{1}(\mathbb{R}, H)$. It may assist the reader to consult [2, 4, 5, 7] for extra details on the approach used here. In particular we consider differentiation as an operator in $H_{\varrho, 0}(\mathbb{R}, H), \varrho \in(0, \infty)$, a weighted $L^{2}$-type space with inner product

$$
\langle\varphi \mid \psi\rangle_{\varrho, 0,0}:=\int_{\mathbb{R}}\langle\varphi(t) \mid \psi(t)\rangle_{0} \exp (-2 \varrho t) d t,
$$

where $\langle\cdot \mid \cdot\rangle_{0}$ denotes the inner product of $H$, which, as a matter of convention, we assume to be linear in the second factor. The resulting operator

$$
\partial_{0}: D\left(\partial_{0}\right) \subseteq H_{\varrho, 0}(\mathbb{R}, H) \rightarrow H_{\varrho, 0}(\mathbb{R}, H)
$$


turns out, [4, 5], to be normal. In particular, $\partial_{0}^{*}=-\partial_{0}+2 \varrho$ and so

$$
\mathfrak{R e} \partial_{0}=\frac{1}{2}\left(\partial_{0}+\partial_{0}^{*}\right)=\varrho .
$$

This observation, or direct computation, gives that for bounded selfadjoint positive $M_{0}: H \rightarrow$ $H$, bounded $M_{1}: H \rightarrow H$ and skew-selfadjoint $A: D(A) \subseteq H \rightarrow H$

$$
\mathfrak{R e}\left\langle u \mid\left(\partial_{0} M_{0}+M_{1}+A\right) u\right\rangle_{\varrho, 0,0}=\left\langle u \mid\left(\varrho M_{0}+\mathfrak{R e} M_{1}\right) u\right\rangle_{\varrho, 0,0}
$$

for $u \in D\left(\partial_{0}\right) \cap D(A)$. With the assumption that

$$
\varrho M_{0}+\mathfrak{R e} M_{1} \geq c_{0}>0
$$

for all sufficiently large $\varrho \in(0, \infty)$, we get that the closure $\overline{\partial_{0} M_{0}+M_{1}+A}$ and its adjoint $\overline{\partial_{0} M_{0}+M_{1}^{*}-A}=\left(\partial_{0} M_{0}+M_{1}+A\right)^{*}$ both have continuous inverses bounded by $\frac{1}{c_{0}}$. In particular, the null space of $\overline{\partial_{0} M_{0}+M_{1}+A}$ and $\left(\partial_{0} M_{0}+M_{1}+A\right)^{*}$ are both trivial. Thus, we have the following well-posedness result, see e.g. [4, 5].

Theorem 2.1 Let $M_{k}: H \rightarrow H, k=0,1$, be continuous linear operators, $M_{0}$ selfadjoint, such that (5) holds for some $c_{0} \in(0, \infty)$ and for all $\varrho \in\left(\varrho_{0}, \infty\right)$ with $\varrho_{0} \in(0, \infty)$ sufficiently large. Moreover let $A: D(A) \subseteq H \rightarrow H$ be skew-selfadjoint. Then

$$
\overline{\partial_{0} M_{0}+M_{1}+A} u=f
$$

has for any $f \in H_{\varrho, 0}(\mathbb{R}, H)$ a unique solution $u \in H_{\varrho, 0}(\mathbb{R}, H)$. Furthermore, $u$ depends on $f$ continuously, i.e.

$$
{\overline{\partial_{0} M_{0}+M_{1}+A}}^{-1}: H_{\varrho, 0}(\mathbb{R}, H) \rightarrow H_{\varrho, 0}(\mathbb{R}, H)
$$

is a continuous linear operator for $\varrho \in\left(\varrho_{0}, \infty\right)$.

As a refinement of (44) we find from integration by parts that for $u \in \stackrel{C}{C}_{1}(\mathbb{R}, H)$ (and so for $\left.u \in D\left(\overline{\partial_{0} M_{0}+M_{1}+A}\right)\right)$ we have

$$
\mathfrak{R e}\left\langle u \mid \chi_{(-\infty, a]}\left(\partial_{0} M_{0}+M_{1}\right) u\right\rangle_{\varrho, 0,0} \geq c_{0}\left\langle\chi_{(-\infty, a]} u \mid \chi_{(-\infty, a]} u\right\rangle_{\varrho, 0,0},
$$

since $|u(a)|^{2} e^{-2 a \varrho} \geq 0$. This yields that we have also causality in the sense of the following theorem.

Theorem 2.2 (Causality) Under the assumptions of Theorem 2.1 we have

$$
\chi_{(-\infty, a]}{\overline{\partial_{0} M_{0}+M_{1}+A}}^{-1}=\chi_{(-\infty, a]}{\overline{\partial_{0} M_{0}+M_{1}+A}}^{-1} \chi_{(-\infty, a]}
$$

for all sufficiently large $\varrho \in(0, \infty)$.

We plan to approach the topic of boundary damping for the Timoshenko beam within this abstract framework, which simplifies matters in so far as we need only ensure that the spatial operator $A$ is skew-selfadjoint and that assumptions of the type (5) hold for $M_{0}, M_{1}$. 


\section{Boundary Damping}

For implementing suitable boundary conditions we consider first a simple example of boundary conditions for the Timoshenko system. Assuming that the beam is described by the unit interval $(-1 / 2,+1 / 2)$, which can always be achieved by translation and re-scaling, following [8] we consider the case of the set of boundary conditions:

$$
\begin{aligned}
V_{1}(\cdot,-1 / 2+0) & =0, \\
V_{1}(\cdot, 1 / 2-0)+c \eta(\cdot, 1 / 2-0) & =0, \\
s(\cdot,-1 / 2+0) & =0 \\
s(\cdot, 1 / 2-0) & =0 .
\end{aligned}
$$

To implement these boundary conditions we consider, in the spirit of abstract grad-div systems as discussed in [6] , the modified system

$$
\left(\partial_{0} M\left(\partial_{0}^{-1}\right)+A\right)\left(\begin{array}{c}
V_{1} \\
\eta \\
\tau_{+} \\
s \\
V_{2}
\end{array}\right)=\left(\begin{array}{c}
0 \\
f_{1} \\
0 \\
f_{2} \\
0
\end{array}\right),
$$

with material law of the simple form

$$
M\left(\partial_{0}^{-1}\right)=M_{0}+\partial_{0}^{-1} M_{1}
$$

with

$$
\begin{aligned}
& M_{0}:=\left(\begin{array}{cccc}
\kappa_{1} & (0,0) & 0 & 0 \\
\left(\begin{array}{l}
0 \\
0
\end{array}\right) & \left(\begin{array}{cc}
\nu_{1} & 0 \\
0 & 0
\end{array}\right) & \left(\begin{array}{l}
0 \\
0
\end{array}\right) & \left(\begin{array}{l}
0 \\
0
\end{array}\right) \\
0 & (0,0) & \nu_{2} & 0 \\
0 & (0,0) & 0 & \kappa_{2}
\end{array}\right) \text {, } \\
& M_{1}:=\left(\begin{array}{cccc}
0 & (0,0) & 0 & 0 \\
\left(\begin{array}{l}
0 \\
0
\end{array}\right) & \left(\begin{array}{ll}
0 & 0 \\
0 & c
\end{array}\right) & \left(\begin{array}{l}
0 \\
0
\end{array}\right) & \left(\begin{array}{l}
1 \\
0 \\
0
\end{array}\right. \\
0 & (0,0) & d & 0 \\
0 & (-1,0) & 0 & 0
\end{array}\right) \text {, }
\end{aligned}
$$

where, we allow $d$ to be a continuous linear operator and $\nu_{1}, \kappa_{1}, \nu_{2}, \kappa_{2}$ continuous selfadjoint and strictly positive definite operators in $L^{2}(-1 / 2,1 / 2)$. For $c: \mathbb{C} \rightarrow \mathbb{C}$ we assume that $c$ is just multiplication by a positive real number. Finally we set $A$ to be the skew-selfadjoint operator

$$
A=\left(\begin{array}{cccc}
0 & B^{*} & 0 & 0 \\
-B & \left(\begin{array}{cc}
0 & 0 \\
0 & 0
\end{array}\right) & \left(\begin{array}{l}
0 \\
0
\end{array}\right) & \left(\begin{array}{c}
0 \\
0
\end{array}\right) \\
0 & (0,0) & 0 & -\partial_{1} \\
0 & (0,0) & -\partial_{1} & 0
\end{array}\right) .
$$


The differentiation operator $\partial_{1}$ denotes the weak derivative in $L^{2}(-1 / 2,1 / 2)$ and the notation $\check{\partial}_{1}$ indicates the use of Dirichlet boundary conditions, which has adjoint $-\partial_{1}$ making $\check{\partial}_{1}$ and $\partial_{1}$ skew-adjoint to each other.

Here we take $B: D(B) \subseteq L^{2}(-1 / 2,1 / 2) \rightarrow L^{2}(-1 / 2,1 / 2) \oplus \mathbb{C} \equiv\left(\begin{array}{c}L^{2}(-1 / 2,1 / 2) \\ \mathbb{C}\end{array}\right)$, where $D(B):=\left\{\varphi \in H_{1}(-1 / 2,1 / 2) \mid \varphi(-1 / 2+0)=0\right\}$, given by

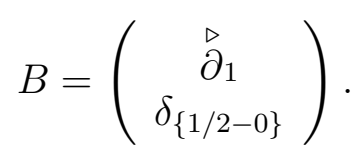

Here $\stackrel{\partial_{1}}{\text { denotes }} \partial_{1}$ with the domain constraint that it only acts on functions in $D(B)$, which in particular vanish at $-1 / 2$.

The 1st, 3rd and 4th boundary conditions follow from the definitions of the domains of $\partial_{1}$ and $\vec{\partial}_{1}$. The first row of this system is the equation

$$
B^{*}\left(\begin{array}{c}
\eta \\
\tau_{+}
\end{array}\right)+\partial_{0}\left(\kappa_{1} V_{1}\right)=0 .
$$

For $u \in D(B)$ with respect to the inner product $\langle\cdot \mid \cdot\rangle$ in $L^{2}(-1 / 2,1 / 2)$ we have

$$
\left\langle u \mid B^{*}\left(\begin{array}{c}
\eta \\
\tau_{+}
\end{array}\right)\right\rangle+\left\langle u \mid \partial_{0}\left(\kappa_{1} V_{1}\right)\right\rangle=0
$$

so

$$
\left\langle B u \mid\left(\begin{array}{c}
\eta \\
\tau_{+}
\end{array}\right)\right\rangle+\left\langle u \mid \partial_{0}\left(\kappa_{1} V_{1}\right)\right\rangle=0
$$

i.e.

$$
\left\langle\left(\begin{array}{c}
\partial_{1} u \\
u(1 / 2-0)
\end{array}\right) \mid\left(\begin{array}{c}
\eta \\
\tau_{+}
\end{array}\right)\right\rangle+\left\langle u \mid \partial_{0}\left(\kappa_{1} V_{1}\right)\right\rangle=0 .
$$

Restricting to $u \in \stackrel{\circ}{H}_{1}(-1 / 2,1 / 2)$ the above equation yields

$$
-\partial_{1} \eta+\partial_{0}\left(\kappa_{1} V_{1}\right)=0 .
$$

Now for $u \in \stackrel{\circ}{H}_{1}(-1 / 2,1 / 2)$ with $u(-1 / 2+0)=0$, integrating by parts we have

$\bar{\tau}_{+} u(1 / 2-0)+\bar{\eta}(\cdot, 1 / 2-0) u(1 / 2-0)-\bar{\eta}(\cdot,-1 / 2+0) u(-1 / 2+0)=\int_{-1 / 2}^{1 / 2} \overline{\left(\partial_{1} \eta-\partial_{0}\left(\kappa_{1} V_{1}\right)\right)} u$

which gives

$$
\tau_{+}+\eta(\cdot, 1 / 2-0)=0 .
$$

The second term of the second row in our system gives

$$
-V_{1}(\cdot, 1 / 2-0)+c \tau_{+}=0
$$


which combined with the previous expression gives

$$
V_{1}(\cdot, 1 / 2-0)+c \eta(\cdot, 1 / 2-0)=0 .
$$

The fact that system (7) falls into the class of abstract operators considered in [2] now yields the following well-posedness theorem.

Theorem 3.1 Let c be a positive real number, $\nu_{1}, \kappa_{1}, \kappa_{2}$ continuous, selfadjoint, strictly positive definite linear operators in $L^{2}(-1 / 2,1 / 2)$. For the operators $\nu_{1}$ and $d$ we require

$$
\varrho \nu_{2}+\mathfrak{R e} d \geq c_{0}>0
$$

for all sufficiently large $\varrho \in(0, \infty)$. Then for any right-hand side $F=\left(0,\left(f_{1}, 0\right), f_{2}, 0\right) \in$ $H_{\varrho, 0}(\mathbb{R}, H)$ with

$$
H=L^{2}(-1 / 2,1 / 2) \oplus\left(L^{2}(-1 / 2,1 / 2) \oplus \mathbb{C}\right) \oplus L^{2}(-1 / 2,1 / 2) \oplus L^{2}(-1 / 2,1 / 2)
$$

there is a unique solution $\left(V_{1},\left(\eta, \tau_{+}\right), s, V_{2}\right) \in H_{\varrho, 0}(\mathbb{R}, H)$.

Remark 3.2 In particular, the boundary conditions (6) are satisfied in the sense of $H_{\varrho,-1}(\mathbb{R}, \mathbb{C})$, which is the space of distributional temporal derivatives of $H_{\varrho, 0}(\mathbb{R}, \mathbb{C})$. Moreover, $F=\left(f_{0},\left(f_{1}, g_{1}\right), f_{2}, f_{3}\right) \in$ $H_{\varrho, 0}(\mathbb{R}, H)$ can be completely arbitrary so that automatically an inhomogeneous boundary condition of the form

$$
V_{1}(\cdot, 1 / 2-0)+c \eta(\cdot, 1 / 2-0)=-g_{1}
$$

can be incorporated. Furthermore, the solution theory extends to data in the space $H_{\varrho,-\infty}(\mathbb{R}, \mathbb{C})$ of finite order distributional temporal derivatives of $H_{\varrho, 0}(\mathbb{R}, \mathbb{C})$, see [2] for details.

\section{Other Material Laws.}

Another special case of boundary damping from the literature, allowing for a non-vanishing right-hand side in [10], is, with adapted names of parameters and variables, given by replacing the second equation of (6) with

$$
\partial_{0} \widetilde{I} \eta(\cdot, 1 / 2-0)+V_{1}(\cdot, 1 / 2-0)+c \eta(\cdot, 1 / 2-0)=-g_{2} .
$$

This amounts to replacing $M_{0}$ in the above with

$$
M_{0}:=\left(\begin{array}{cccc}
\kappa_{1} & (0,0) & 0 & 0 \\
\left(\begin{array}{l}
0 \\
0
\end{array}\right) & \left(\begin{array}{cc}
\nu_{1} & 0 \\
0 & \widetilde{I}
\end{array}\right) & \left(\begin{array}{l}
0 \\
0
\end{array}\right) & \left(\begin{array}{l}
0 \\
0
\end{array}\right) \\
0 & (0,0) & \nu_{2} & 0 \\
0 & (0,0) & 0 & \kappa_{2}
\end{array}\right) .
$$

The parameter $c$ along with the additional parameter $\widetilde{I}$ are now allowed to be non-negative reals with not both zero. In regards to the model equations used in [8], which differ slightly 
from the above system of partial differential equations for the Timoshenko beam, it may be advisable to consider the more general variant

$$
M_{1}:=\left(\begin{array}{cccc}
0 & (0,0) & 0 \\
\left(\begin{array}{l}
0 \\
0
\end{array}\right) & \left(\begin{array}{cc}
0 & 0 \\
0 & c
\end{array}\right) & \left(\begin{array}{l}
0 \\
0
\end{array}\right) & \left(\begin{array}{c}
-\sigma_{0}^{*} \\
0 \\
0
\end{array}\right. \\
0 & (0,0) & d & 0 \\
0 & \left(\sigma_{0}, 0\right) & 0 & 0
\end{array}\right)
$$

with a non-vanishing number (or operator) $\sigma_{0}$. It may be noteworthy that for $d=0=c$ we have a system in which the norm of

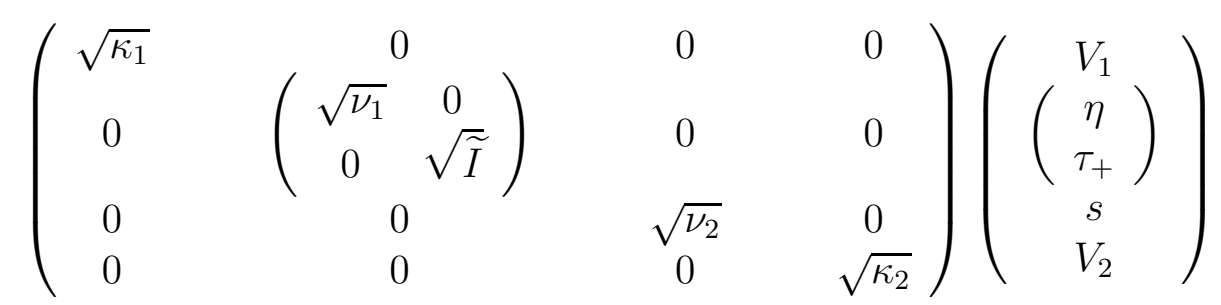

is conserved, assuming a pure initial value problem. We remark that in some model equations in the literature the rotational displacement enters with the opposite sign. This is, however, just a unitarily congruent version of our system obtained via the unitary transformation matrix

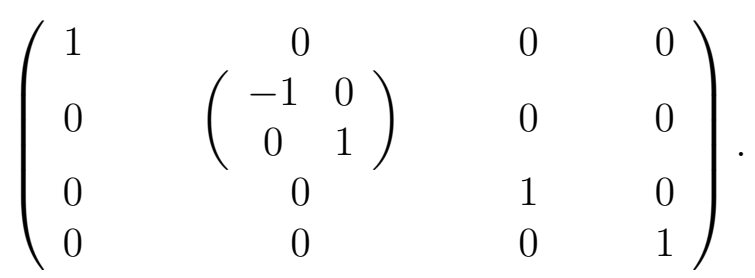

This transformation changes only $B$ and $M_{1}$ slightly to

$$
B=\left(\begin{array}{c}
-\stackrel{\triangleright}{\partial}_{1} \\
\delta_{\{1 / 2-0\}}
\end{array}\right)
$$

and

$$
M_{1}:=\left(\begin{array}{cccc}
0 & (0,0) & 0 & 0 \\
\left(\begin{array}{l}
0 \\
0
\end{array}\right) & \left(\begin{array}{cc}
0 & 0 \\
0 & c
\end{array}\right) & \left(\begin{array}{l}
0 \\
0
\end{array}\right) & \left(\begin{array}{c}
\sigma_{0}^{*} \\
0 \\
0
\end{array}\right. \\
0 & (0,0) & d & 0 \\
0 & \left(-\sigma_{0}, 0\right) & 0 & 0
\end{array}\right)
$$

Indeed, completely general material law operators $M\left(\partial_{0}^{-1}\right)$ can be handled in the same way, where $M$ is a bounded operator-valued function, analytic in a ball of positive radius $r$ around the point $r$ on the real axis. The crucial assumption is that the numerical range of 
$\partial_{0} M\left(\partial_{0}^{-1}\right)$ is in the right half plane and uniformly bounded away from the imaginary axis for all sufficiently large $\varrho \in(0, \infty)$ :

$$
\mathfrak{R e}\left\langle u \mid \partial_{0} M\left(\partial_{0}^{-1}\right) u\right\rangle_{\varrho, 0,0} \geq c_{0}\langle u \mid u\rangle_{\varrho, 0,0}
$$

for some $c_{0} \in(0, \infty)$ and all $u \in D\left(\partial_{0}\right)$. This allows for a number of more intricate coupling phenomena.

If we assume that

$$
M\left(\partial_{0}^{-1}\right)=\left(\begin{array}{cccc}
M_{00}\left(\partial_{0}^{-1}\right) & M_{01}\left(\partial_{0}^{-1}\right) & 0 & 0 \\
M_{10}\left(\partial_{0}^{-1}\right) & M_{11}\left(\partial_{0}^{-1}\right) & 0 & 0 \\
0 & 0 & M_{22}\left(\partial_{0}^{-1}\right) & M_{23}\left(\partial_{0}^{-1}\right) \\
0 & 0 & M_{32}\left(\partial_{0}^{-1}\right) & M_{33}\left(\partial_{0}^{-1}\right)
\end{array}\right)
$$

the system actually decomposes 1 into two $2 \times 2$-systems. Focusing on the first block system we get

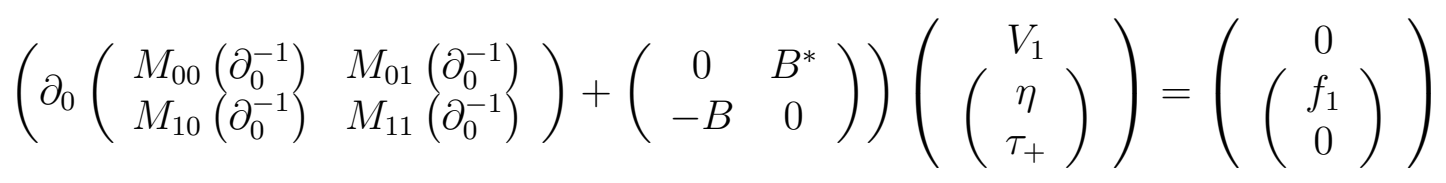

where $B$ is as in (8) .

If we take

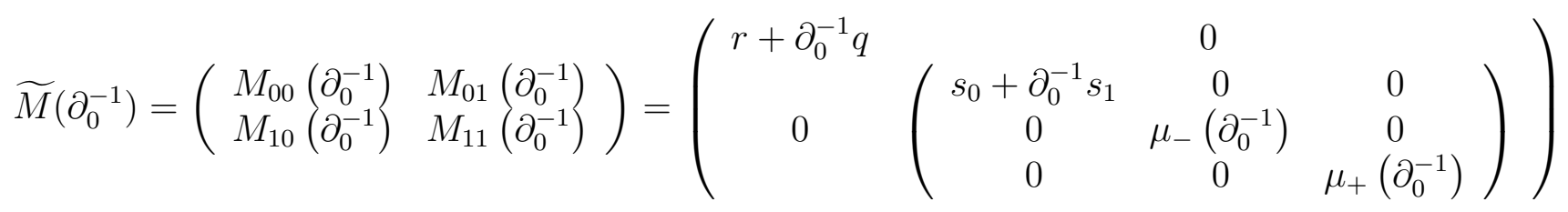

then for $s_{0}=p^{-1}$ and $s_{1}=0$ we obtain the undamped hyperbolic case, while for $s_{0}=0$ and $s_{1}=p^{-1}$ we have the parabolic case of the first order formulation of time-dependent Sturm-Liouville problems (in standard terms). Here we replace $B$ by

$$
\widetilde{B}=\left(\begin{array}{c}
\partial_{1} \\
\delta_{\{-1 / 2+0\}} \\
\delta_{\{1 / 2-0\}}
\end{array}\right)
$$

The resulting operator equation

$$
\left.\left(\partial_{0} \widetilde{M}\left(\partial_{0}^{-1}\right)+\left(\begin{array}{cc}
0 & B^{*} \\
-B & 0
\end{array}\right)\right)\left(\left(\begin{array}{c}
V_{1} \\
\tau_{+} \\
\tau_{-}
\end{array}\right)\right)=\left(\begin{array}{c}
0 \\
f_{1} \\
0 \\
0
\end{array}\right)\right)
$$

\footnotetext{
${ }^{1}$ Indeed, the coupling of all four equations is due to the off-diagonal entries in $\mathcal{M}_{1}$ of the original first order Timoshenko beam model.
} 
results in the first two boundary conditions of (6) being replaced by dynamic boundary conditions

$$
\begin{array}{r}
\partial_{0} \mu_{+}\left(\partial_{0}^{-1}\right) \eta(\cdot, 1 / 2-0)+V_{1}(\cdot, 1 / 2-0)=0 \\
\partial_{0} \mu_{-}\left(\partial_{0}^{-1}\right) \eta(\cdot,-1 / 2+0)-V_{1}(\cdot,-1 / 2+0)=0
\end{array}
$$

where $\mu_{+}, \mu_{-}$satisfy the assumption stated for $M$ in (9). The point-wise evaluated FourierLaplace transformed system (i.e. replacing $\partial_{0}$ by $z=i \lambda+\varrho$ ) is discussed in [1, 9] for the parabolic case, i.e. $s_{0}=0$, where $R_{1}(z):=z \mu_{+}\left(\frac{1}{z}\right), R_{0}(z):=z \mu_{-}\left(\frac{1}{z}\right)$ define rational Nevanlinna functions $R_{0}$ and $R_{1}$.

\section{Other boundary conditions.}

A variety of other boundary conditions are also accessible via the above approach. For example, implementing dynamic boundary conditions in all unknowns and for both boundary parts we replace $A$ with

$$
\widetilde{A}=\left(\begin{array}{cccc}
0 & \widetilde{B}^{*} & 0 & 0 \\
-\widetilde{B} & 0 & 0 & 0 \\
0 & 0 & 0 & \widetilde{B}^{*} \\
0 & 0 & -\widetilde{B} & 0
\end{array}\right)
$$

The system now becomes

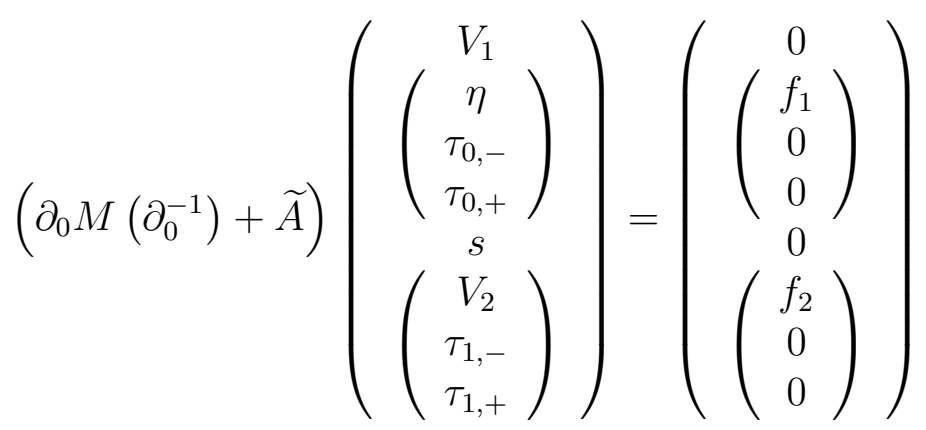

If here again we assume that

$$
M\left(\partial_{0}^{-1}\right)=\left(\begin{array}{cccc}
M_{00}\left(\partial_{0}^{-1}\right) & M_{01}\left(\partial_{0}^{-1}\right) & 0 & 0 \\
M_{10}\left(\partial_{0}^{-1}\right) & M_{11}\left(\partial_{0}^{-1}\right) & 0 & 0 \\
0 & 0 & M_{22}\left(\partial_{0}^{-1}\right) & M_{23}\left(\partial_{0}^{-1}\right) \\
0 & 0 & M_{32}\left(\partial_{0}^{-1}\right) & M_{33}\left(\partial_{0}^{-1}\right)
\end{array}\right)
$$

also this system decouples into two $2 \times 2$-systems. Focusing on the first block system we get

$$
\left.\left(\partial_{0} \widetilde{M}\left(\partial_{0}^{-1}\right)+\left(\begin{array}{cc}
0 & \widetilde{B}^{*} \\
-\widetilde{B} & 0
\end{array}\right)\right)\left(\left(\begin{array}{c}
V_{1} \\
\tau_{-} \\
\tau_{+}
\end{array}\right)\right)=\left(\begin{array}{l}
0 \\
f \\
0 \\
0
\end{array}\right)\right)
$$


which results in dynamic boundary conditions as noted in the previous section, likewise with the Sturm-Liouville case being a particular application.

\section{References}

[1] P. A. Binding, P. J. Browne, B. A. Watson, Sturm-Liouville problems with boundary conditions rationally dependent on the eigenparameter, II, J. Comp. Applied Math., 148:147$168,2002$.

[2] R. Picard. A Structural Observation for Linear Material Laws in Classical Mathematical Physics. Math. Methods Appl. Sci., 32(14):1768-1803, 2009.

[3] R. Picard. Mother Operators and their Descendants. Journal of Mathematical Analysis and Applications, 403(1):54-62, 2013.

[4] R. Picard. An Elementary Hilbert Space Approach to Evolutionary Partial Differential Equations. Rend. Istit. Mat. Univ. Trieste, 42 suppl.:185-204, 2010.

[5] R. Picard and D. F. McGhee. Partial Differential Equations: A unified Hilbert Space Approach, volume 55 of De Gruyter Expositions in Mathematics. De Gruyter. Berlin, New York. 518 p., 2011.

[6] Rainer Picard, Stefan Seidler, Sascha Trostorff, and Marcus Waurick. On abstract graddiv systems. Journal of Differential Equations, 260(6):4888 - 4917, 2016.

[7] R. Picard and B.A. Watson Evoluationary problems involving Sturm-Liouville operators. Operator Theory: Advance and Applications, 236:393-406, 2013.

[8] A. Roux, A.J. van der Merwe, and N.F.J. van Rensburg. Elastic waves in a Timoshenko beam with boundary damping. Wave Motion, 57:194 - 206, 2015.

[9] E. M. Russakovskii, The matrix Sturm-Liouville problem with spectral parameter in the boundary conditions. Algebraic and operator aspects, Trans. Moscow Math. Soc., 57 (1996), 159-184.

[10] L. Zietsman, N.F.J. van Rensburg, and A.J. van der Merwe. A Timoshenko beam with tip body and boundary damping. Wave Motion, 39(3):199 - 211, 2004. 